EPJ Web of Conferences 16, 06004 (2011)

DOI: $10.1051 /$ epjconf/20111606004

(C) Owned by the authors, published by EDP Sciences, 2011

\title{
A search for southern ultracool dwarfs in young moving groups
}

\author{
J.R.A. Clarke ${ }^{1, a}$, D.J. Pinfield ${ }^{1}$, M.C. Galvez-Ortiz"1, J.S. Jenkins ${ }^{2}$, \\ B. Burningham ${ }^{1}$, N.R. Deacon ${ }^{3,4}$, H.R.A. Jones ${ }^{1}$, R.S. Pokorny ${ }^{5}$, \\ J.R. Barnes ${ }^{1}$ and A.C. Day-Jones ${ }^{1,2}$ \\ 1 Centre For Astrophysics Research, University of Hertfordshire, College Lane, Hatfield, \\ Hertfordshire, AL10 9AB, UK \\ 2 Department of Astronomy, Universidad de Chile, Casilla Postal 36D, Santiago, Chile \\ 3 Department of Astrophysics, Faculty of Science, Radboud University Nijmegen, \\ PO Box 9010, 6500 GL Nijmegen, The Netherlands \\ 4 Institute for Astronomy, University of Hawaii, 2680 Woodlawn Drive, Honolulu, \\ HI 96822, USA \\ 5 Yunnan Observatory, PO Box 110, CAS, 650011 Kunming, P.R. China
}

\begin{abstract}
We have constructed an 800-strong red object catalogue by cross-referencing optical and infrared catalogues with an extensive proper motion catalogue compiled for red objects in the southern sky to obtain proper motions. We have applied astrometric and photometric constraints to the catalogue in order to select ultracool dwarf moving group candidates. 132 objects were found to be candidates of a moving group. From this candidate list we present initial results. Using spectroscopy we have obtained reliable spectral types and space motions, and by association with moving groups we can infer an age and composition. the further study of the remainder of our candidates will provide a large sample of young brown dwarfs and confirmed members will provide benchmark ultracool dwarfs. These will make suitable targets of AO planet searches.
\end{abstract}

\section{INTRODUCTION}

Moving group (MG) populations are distinguishable from the field by their astrometric properties. A MG can remain kinematically distinct within the general field population at ages up to $\sim 1$ Gyr [1]. MG populations are thought to originate in the same formation environment as open clusters and therefore consist of young populations with characteristic space motions, thus membership of such groups can be used to constrain the age and composition of members. A variety of studies have shown that in general, the younger MGs are consistent with coeval populations of uniform metallicity (e.g. $[3,4])$.

The identification of ultracool dwarfs (spectral type later the $~ M 6$ ) in MGs facilitates a variety of interesting areas of astronomical study. Ultracool MG members will provide excellent test-beds (with known age and metallicity) with which to improve our theoretical understanding of low temperature and low gravity dusty-atmospheres. Nearby intrinsically faint young stars facilitate particularly sensitive searches for binary companions over a large range of separation and mass-ratios. Close low-mass binary systems in MGs could yield dynamical mass measurements, which could be used to test evolutionary models of low-mass stars and substellar objects [5]. Also, the youth and potential proximity of MG members could also allow one to search for planetary companions (e.g. [6]) via adaptive optics (AO) techniques (e.g. [7]).

\footnotetext{
ae-mail: j.r.a.clarke@herts.ac.uk
}

This is an Open Access article distributed under the terms of the Creative Commons Attribution-Noncommercial License 3.0, which permits unrestricted use, distribution, and reproduction in any noncommercial medium, provided the original work is properly cited. 


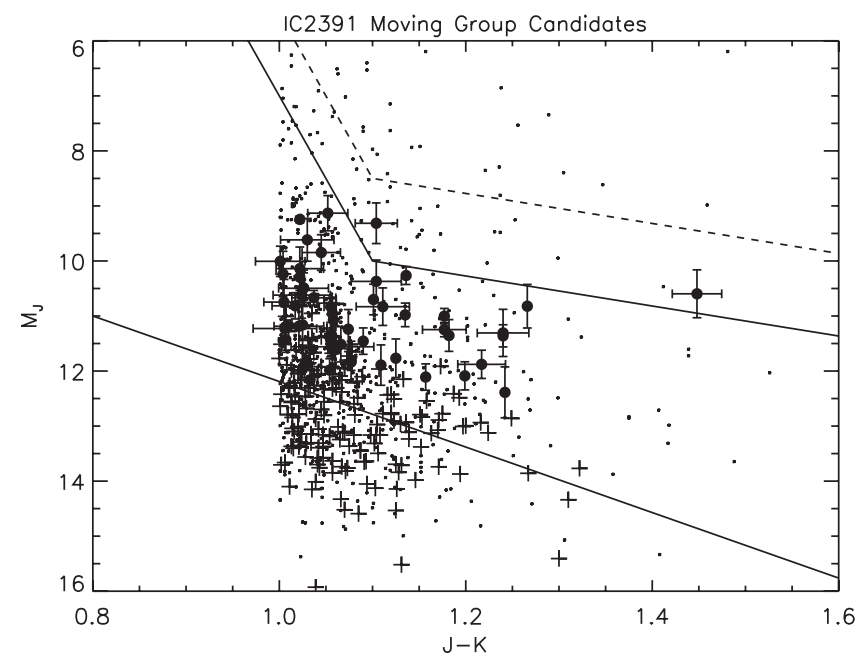

Figure 1. An example $\mathrm{M}_{\mathrm{J}}$, J-K CMD selection for the IC2391 MG. The expected location of the dwarf sequence is enclosed by solid lines with the alternative upper boundary (for young sources) shown as a dashed line. Catalogue objects are shown as points. Objects passing the astrometric selection (see Section 3.2) are shown as plus signs. Objects also passing our photometric selection are shown as filled circles.

\section{CREATING THE MOVING GROUP CANDIDATES}

We created a red object high-proper motion catalogue using an extended version of the LiverpoolEdinburgh High Proper Motion Catalogue and Southern Infrared Proper Motion Survey. Cross matching with 2MASS using colour cuts requiring $\mathrm{J}-\mathrm{K}_{s} \geq 1.0$ and $\mathrm{R}-\mathrm{K}_{s} \geq 5.0$ to ensure objects with a spectral type predominantly later than M6 were returned (e.g. [8]). In total, our final high proper motion red object catalogue consisted of 817 objects with near infrared photometry.

\subsection{Astrometric Selection}

We considered five MGs (the Pleiades, Hyades, Sirius, IC2391 and Castor MGs; aged 50-600 Myr) and were able to identify catalogue objects whose proper motion was orientated towards convergent points (points on the sky with towards which all MG members move towards) of these MGs. Derivations of [9] allowed us to select objects that had comparatively small proper motion perpendicular to the direction of the convergent point, allowing for a scatter of $\pm 5 \mathrm{~km} \mathrm{~s}^{-1}$ calculated using an astrometrically estimated distance (also from [9]).

\subsection{Photometric selection}

To assess if an object's colour magnitude diagram (CMD) location is consistent we defined a dwarf sequence on two CMDs using samples of canonical objects covering the full colour (and spectral type) range of our catalogue objects, but with distances from parallax measurements. For this purpose we used known $\mathrm{M}$ and $\mathrm{L}$ dwarf samples. I, J and K-band photometry was available for all our red catalogue objects, so $\mathrm{M}_{\mathrm{J}}$ versus I-J and $\mathrm{M}_{\mathrm{J}}$ versus J-K CMDs could thus be created using the astrometric distance to photometrically test those that passed the astrometric selection criteria.

By applying both the astrometric and photometric tests to the red object catalogue we identified 132 objects that are candidate members of the five MGs. Figure 1 shows an example $\mathrm{M}_{\mathrm{J}}$ versus J-K CMD for the IC2391 MG. Solid lines show the limits of the dwarf sequence, with the dotted line representing the brighter limit corrected for youth. Objects that passed only the astrometric test are plotted as plus signs and those that path both tests are plotted as filled circles. 


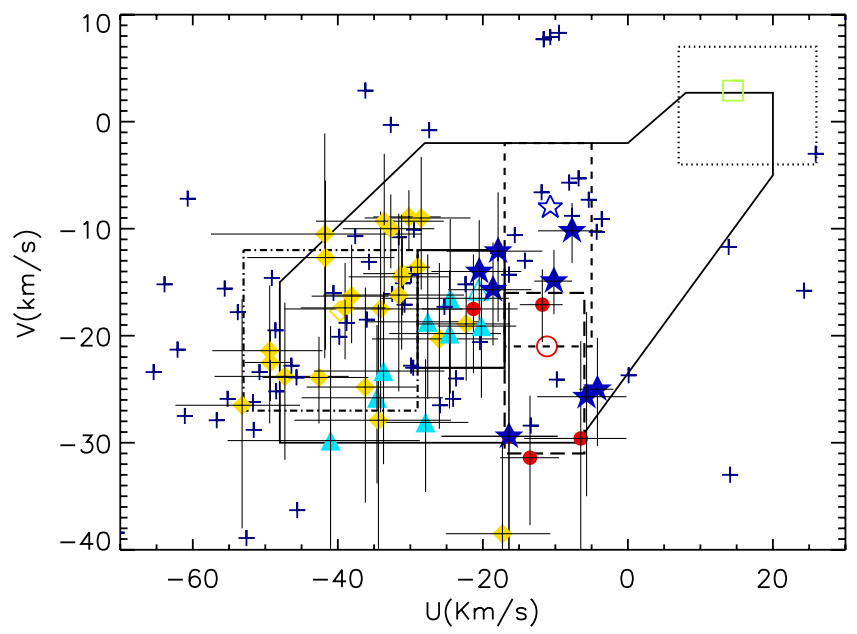

Figure 2. $V$ versus $U$ space motions of the $70 \mathrm{MG}$ candidates with radial velocities from our observations with FEROS, UVES, Gemini and from the literature plotted as symbols corresponding to their kinematic membership. Kinematic members are plotted as filled diamonds, triangles, circles, stars and, squares for the Hyades, IC2391, Pleiades, Castor and Sirius MGs respectively. Non-kinematic members are plotted as blue crosses. The solid outline represents the region of velocity space that young-disk objects are found.

\section{GALACTIC SPACE MOTIONS}

To further constrain the MG membership of the 132 candidate objects it was necessary to calculate galactic space motions from proper motions and radial velocities. To provide us with radial velocities we obtained and reduced high-resolution spectra of $\sim 70$ objects using FEROS on the $2.2 \mathrm{~m}$ telescope at La Silla Chile, UVES on the VLT and Phoenix at Gemini south.

We cross-correlated several orders of the reduced spectra using an IDL routine and FXCOR in IRAF. A heliocentric correction was made to calculate radial velocities with respect to the Sun. Using these radial velocities we derived space motions for these objects using the transformation matrices of [10], correcting for youth due to potential group membership where applicable.

To assess the kinematic MG membership of these objects, we compared their kinematics with the measured space motions of higher mass MG members from the literature $[4,11]$. In this way we were able to effectively define the space motion ranges that are representative of each MG. Figure 2 shows these limits and the space motions of the $\sim 70$ radial velocity objects in $V U$ velocity space. The MGs' space motion represented by hollow symbols with a diamond, triangle, circle, star and square for the Hyades, IC2391, Pleiades, Castor and Sirius MGs respectively. Kinematic members of these MGs are plotted as filled symbols corresponding to their kinematic group membership and objects that are not kinematic members are plotted as blue crosses. These kinematic members have also passed the kinematic test in $W V$ velocity space. From this we have 32 candidates that are kinematically consistent with MG membership. We find 22, 8, 8 and 4 kinematic members of the Hyades, IC2391, Castor and Pleiades MGs respectively. These results can also be found in [12].

\section{KINEMATIC MEMBER FOLLOW UP}

Figure 3 shows a plot of age (based upon potential group membership) versus spectral type (based upon $\mathrm{M}_{\mathrm{J}}$ versus Spectral type relations from [13] for our candidates). The figure illustrates which objects would be selected for the various methods of follow up to confirm youth and thus finalising membership. Candidates that appear to the right of the lithium line can be followed up with a lithium test programme (51 objects). There are also 51 candidates that appear younger than $200 \mathrm{Myr}$ so are eligible for follow 


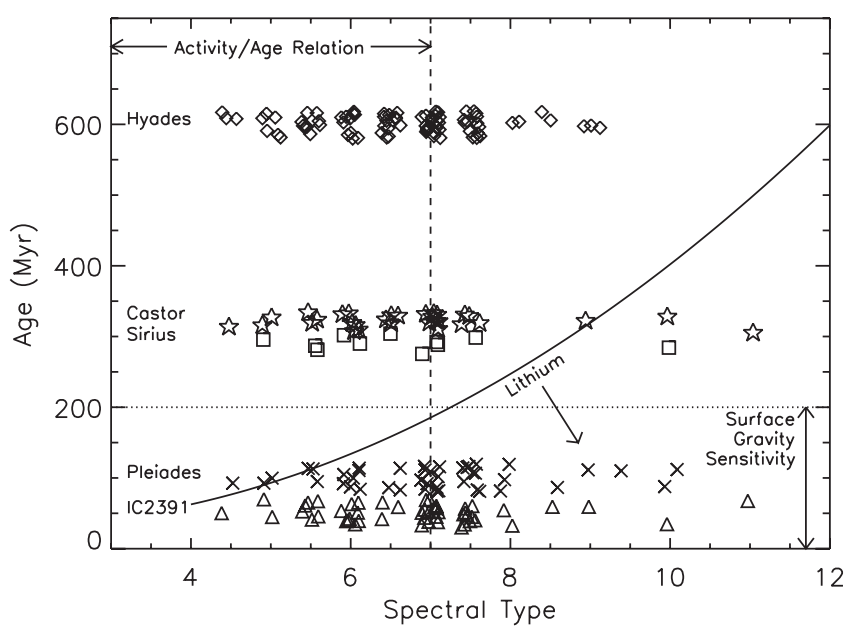

Figure 3. Figure showing age versus spectral type for the candidates along with approximate follow-up limits. The solid line marks the lithium edge with lithium depleted objects to the left. The dashed line marks the limit of age/activity relation studies where objects to the left are plausible for testing. The dotted line represents the upper age limit of testing for MG membership by gravity sensitive spectral features. We plot members of the Castor, Sirius, Pleiades, IC2391 and Hyades MGs as stars, squares, crosses, triangles and diamonds respectively.

up using spectroscopic gravity sensitive features. We have 93 candidates left of the spectral type $=$ M7 limit and would thus be suitable for age/activity relation follow up. Of our complete candidate list we have 23 objects that cannot be tested by any of these methods but will be eligible for age testing using rotational velocities. Rotational velocities could also be used as an extra age constraint for the whole of our candidate list (see [14]). Thus, using spectroscopic methods we will apply at least one non-kinematic test to all our MG candidate providing a solid sample of MG members. Kinematically confirmed members could also provide a substantial sample of targets for AO imaging. For details see Galvéz et al. in these proceedings.

\section{References}

[1] De Simone R., Xiaoan W., Tremaine S., 2004, MNRAS, 350, 627

[2] Eggen O. J., 1958, MNRAS, 118, 65

[3] Soderblom D. R., Mayor M., 1993, ApJ, 105, 226

[4] Montes D., López-Santiago J., Gálvez M. C., Fernandez-Figueroa M. J., De Castro E., Cornide M., 2001, MNRAS, 328, 45

[5] Pinfield D. J., Jones H. R. A., Steele I. A., 2005, PASP, 117, 173

[6] Chauvin G. et al., 2004, A\&A, 425, L29

[7] Biller B. A. et al., 2006, ApJ, 647, 464

[8] Kirkpatrick J. D et al., 1999, ApJ, 519, 802

[9] Reid I. N., et al 1999, ApJ, 521, 613

[10] Johnson D. R. H., Soderblom D. R., 1987, AJ, 93, 864

[11] Barrado Y Navacués D., 2006, A\&A, 459, 511

[12] Clarke J. R. A., et al., 2009, preprint (arXiv:0805.4772)

[13] Dahn C. C. et al., 2002, AJ, 124, 1170

[14] Jenkins J. S., Ramsey L. W., Jones H. R. A., Pavlenko Y., Gallardo J., Barnes J. R., Pinfield D. J., 2009, ApJ, 704, 975 